\title{
Determination of the blood oxygenation in the brain by time- resolved reflectance spectroscopy: contribution of vascular absorption and tissue background absorption
}

Andreas H. Hielscher, Hanli Liu, Lihong V. Wang, Frank K. Tittel, Britton Chance, et al.

Andreas H. Hielscher, Hanli Liu, Lihong V. Wang, Frank K. Tittel, Britton Chance, Steven $\mathrm{L}$. Jacques, "Determination of the blood oxygenation in the brain by time-resolved reflectance spectroscopy: contribution of vascular absorption and tissue background absorption," Proc. SPIE 2136, Biochemical Diagnostic Instrumentation, (21 July 1994); doi: 10.1117/12.180773

SPIE. Event: OE/LASE '94, 1994, Los Angeles, CA, United States 


\title{
Determination of blood oxygenation in the brain \\ by time resolved reflectance spectroscopy (I) : \\ Influence of the skin, skull, and meninges
}

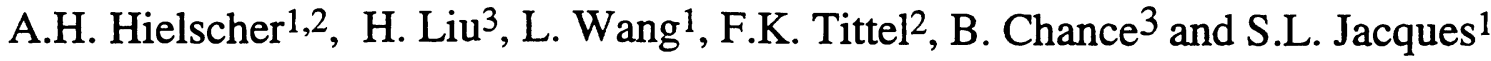 \\ 1 University of Texas M.D. Anderson Cancer Center, \\ Laser Biology Research Laboratory, Houston, Texas 77030 \\ 2Rice University, Dept. of Electrical and Computer Engineering, \\ Houston, Texas 77251-1892 \\ 3University of Pennsylvania, Dept. of Biochemistry and Biophysics, \\ Philadelphia, Pennsylvania 19104-6089
}

\begin{abstract}
In recent years the possibility of measuring the blood oxygenation in the brain with near infrared light has been studied by several authors. However the fact that the brain is encapsulated by different layers of tissues (skin, skull, meninges) has attracted little attention. The goal of this study was to quantify the influence of these different layers on brain blood oxygenation measurements. Experimental results obtained from time resolved reflectance measurements on layered tissue phantoms were compared to Monte Carlo simulations of layered models, diffusion theory, and in vivo measurements on the human head.

Both, the experimental results and simulations show that the absorption coefficient $\mu_{\mathrm{a}}$, which is closely related to the blood oxygenation, of deeper layers can be accessed in the time domain. The early parts ( $\mathrm{t} \leq 1.0 \mathrm{~ns}$ ) of the impulse response are influenced by the outer layers of the head (skin, skull, meninges), which leads to a shift of the peak position of the impulse response. However the later parts ( $t \geq 1.0 \mathrm{~ns})$ are clearly dominated by the optical properties of the underlying tissue. Thus by fitting analytical expressions found from diffusion theory only to the late part of the time resolved reflectance allows to determine $\mu_{\mathrm{a}}$ and subsequently the blood oxygenation of the deepest medium (e.g. brain tissue).

\section{INTRODUCTION}

Two major areas, where continuously monitoring the blood oxygenation in the brain is of major interest are newborn and cardiac surgery intensive care. Cerebral injuries due to hypoxia as well as hyperoxia are considered to be the cause of about one third of all deaths in the full term infants ${ }^{1}$. In the preterm infant it is estimated to be the cause of neuro-developmental abnormality in up to two thirds of the survivors ${ }^{2}$. Of the 340,000 patients who undergo cardiac surgery each year in the US, roughly 100,000 suffer at least a minor neurological deficit because of cerebral ischemia, during or after surgery. Therefore objective measurement techniques are needed which allow for an oxygen support that is sufficient yet minimal toxic.
\end{abstract}


The clinical symptoms of hypoxia (e.g. tachypnea, tachycardia or cyanosis) and hyperoxia (e.g. worsening lung disease from direct oxygen toxicity or retinopathy) are unreliable indicators and may even be completely absent until irreversible damage has occurred 3 . The same critique holds for conventional imaging methods like ultrasound, X-ray, CT or NMR, which can only detect irreversible tissue damages ${ }^{4}$. The "gold standard" assays, arterial blood gas measurement or estimation of oxygen consumption, are invasive, expensive, often painful, and risky. In addition, these standard methods only reveal static information about oxygenation at one point in time, rather than continuous measurements over time. What is needed is a technique for non invasively and continuously monitoring the oxygenation status of the blood in the brain. This would allow to intervene in time to prevent irreversible damage.

This paper first discusses briefly the possibilities and limitations of a current state of the art method of non invasively measuring the blood oxygenation with light. This will be followed by a proposal how to overcome these limitations with time resolved reflectance measurements. After an introduction to the principles of these techniques, the problem of layered tissue structures will be addressed in detail. The brain is knowingly encapsulated by different layers with varying optical properties (skin, skull and meninges). How this layers effect the determination of the blood oxygenation of the underlying brain tissue, the brain, is of substantial interest and has up to know not been studied systematically.

\section{STATE OF THE ART TECHNIQUE}

Jöbsis first demonstrated the possibility of detecting near infrared light of only $48 \mu \mathrm{Wcm}^{-2}$ in a $6.6 \mathrm{~nm}$ wide band around $800 \mathrm{~nm}$, after it traveled through a brain of $13.3 \mathrm{~cm}$ diameter 5 . In the wavelength region between 700-900 nm, known as the therapeutical window, the major absorbing chromophores in the brain are the two blood constituents hemoglobin $(\mathrm{Hb})$ and oxygenated hemoglobin $\left(\mathrm{HbO}_{2}\right)$. These two absorbers display well known different absorption characteristics 6 . Therefore, with the use of at least 2 wavelength it is in principle possible to determine the two unknowns, the concentration of hemoglobin [ $\mathrm{Hb}]$ and deoxygenated hemoglobin $\left[\mathrm{HbO}_{2}\right]$, with a set of linear equations:

$$
\begin{aligned}
& \mathrm{A}^{\lambda 1}=\left\{\varepsilon_{\mathrm{Hb}}^{\lambda 1}[\mathrm{Hb}]+\varepsilon_{\mathrm{HbO}_{2}}^{\lambda_{1}}\left[\mathrm{HbO}_{2}\right]\right\} \mathrm{L}^{\lambda 1} \\
& \mathrm{~A}^{\lambda 2}=\left\{\varepsilon_{\mathrm{Hb}}^{\lambda_{2}}[\mathrm{Hb}]+\varepsilon_{\mathrm{HbO}_{2}}^{\lambda_{2}}\left[\mathrm{HbO}_{2}\right]\right\} \mathrm{L}^{\lambda 2}
\end{aligned}
$$

where

$$
\begin{aligned}
\mathrm{A}^{\lambda} & :=\text { Absorbance at wavelength } \lambda . \\
\varepsilon & :=\text { Extinction coefficient }\left[\mathrm{mM}^{-1} \mathrm{~cm}^{-1}\right] \\
\mathrm{L}^{\lambda} & :=\text { Optical path length at wavelength } \lambda .
\end{aligned}
$$

Several studies have shown the possibility of monitoring changes in the blood oxygenation based on the equations $(1 \mathrm{a}, \mathrm{b}){ }^{4-9}$. However in order to quantify [Hb] and $\left[\mathrm{HbO}_{2}\right]$ from the measurements of the absorbance (A), the path length (L) traveled by the photons before they reach the detector must be known. Usually $L$ is assumed to be a constant, independent of wavelength, blood concentration, or individuals. However Benaron et. al found that the path lengths measured among infants of even similar weight and age differ substantially 10 . Since blood itself is a highly 
scattering medium, with a different scattering coefficient then brain 11,12 , the total blood volume $\mathrm{V}_{\text {tot }}=[\mathrm{Hb}]+\left[\mathrm{HbO}_{2}\right]$ will also affect the path length. Thus devices based on pure absorbance measurements may only qualitatively detect changes of the oxygenation status, but fail to give absolute blood volume and oxygenation values. To solve this problem one has to measure the absorption coefficient $\mu_{\mathrm{a}}$, which is the absorbance $\mathrm{A}$ per unit pathlength.

\section{TIME RESOLVED REFLECTANCE SPECTROSCOPY}

Time resolved reflectance measurements on tissues can provide the necessary information for the determination of the absorption coefficient $\mu_{\mathrm{a}}$ of tissues. As will be demonstrated in this paper, this method is especially suited for the determination of the absorption coefficient of the brain which is encapsulated by layers of tissues with different optical properties. In this technique a picosecond pulsed light source is used as an input signal and the reflectance is measured as a function of time at a distance of a few centimeter. A detailed description of the experimental set up can be found elsewhere ${ }^{13}$. In this work the correct interpretation of the measured data, will be discussed. First a semi-infinite homogenous media is studied. It will be shown that the results found for this simple case, can be generalized to the more complicated problem of layered tissue structures. This will illuminate the influence of the skin, skull and meninges, on blood oxygenation measurements of the brain. In part II of this study 14 on blood oxygenation measurements of the brain, the consideration will be extended to the case of distributed local absorbers (blood in vessels) embedded in a homogenous medium.

\subsection{Homogenous Tissues}

Currently there exist essentially two methods to extract $\mu_{\mathrm{a}}$ from a time resolved reflectance measurement. The most accurate, but also the most computation time consuming one is based on Monte Carlo simulations. In this kind of computer simulations light is treated as consisting of single photons, which are injected into the tissue one by one. Each single photon bounces around statistically inside the tissue, until it is either absorbed or reemitted from the medium. The statistics of the photon propagation process depends on the absorption and scattering coefficient of the tissue. These coefficients basically describes the probability that a photon gets either absorbed or scattered over a unit length. A comprehensive description of Monte Carlo simulation techniques can be found in [15] and [16].

If a large enough number of photons $\left(\sim 10^{6}\right)$ is injected into a tissue with given optical properties, Monte Carlo simulations have been found to give rather accurate description of the light distribution inside and outside the tissue. Figure 1 shows a comparison of an experimental result and a Monte Carlo simulation. The experiment was performed on a homogenous tissue phantom, made out of gelatin with polystyrene spheres which act as scatterers and india ink which provides the absorption. From the concentration of polystyrene spheres and india ink one can calculate the expected optical properties. These properties were fed into the Monte Carlo code developed in our laboratory. The result of the simulation was compared to the measurement. The difference between the Monte Carlo simulation and experiment was minimized by changing the optical input parameters for the simulation slightly and rerun the program. The final result of the

optical properties determined by the Monte Carlo simulation in this case was $5 \%$ above the 
calculated ones. This is within the error for the calculations, since they depend on the exact concentration measurement of polystyrene spheres and india ink. As already mentioned, the drawback of Monte Carlo simulations is that they take a long time for completion. In the described example each simulation with $2,000,000$ photons took about 30 hours on a sparc10workstation. This is much too long for any practical algorithm for the determination of blood oxygenation applied in a clinical environment.

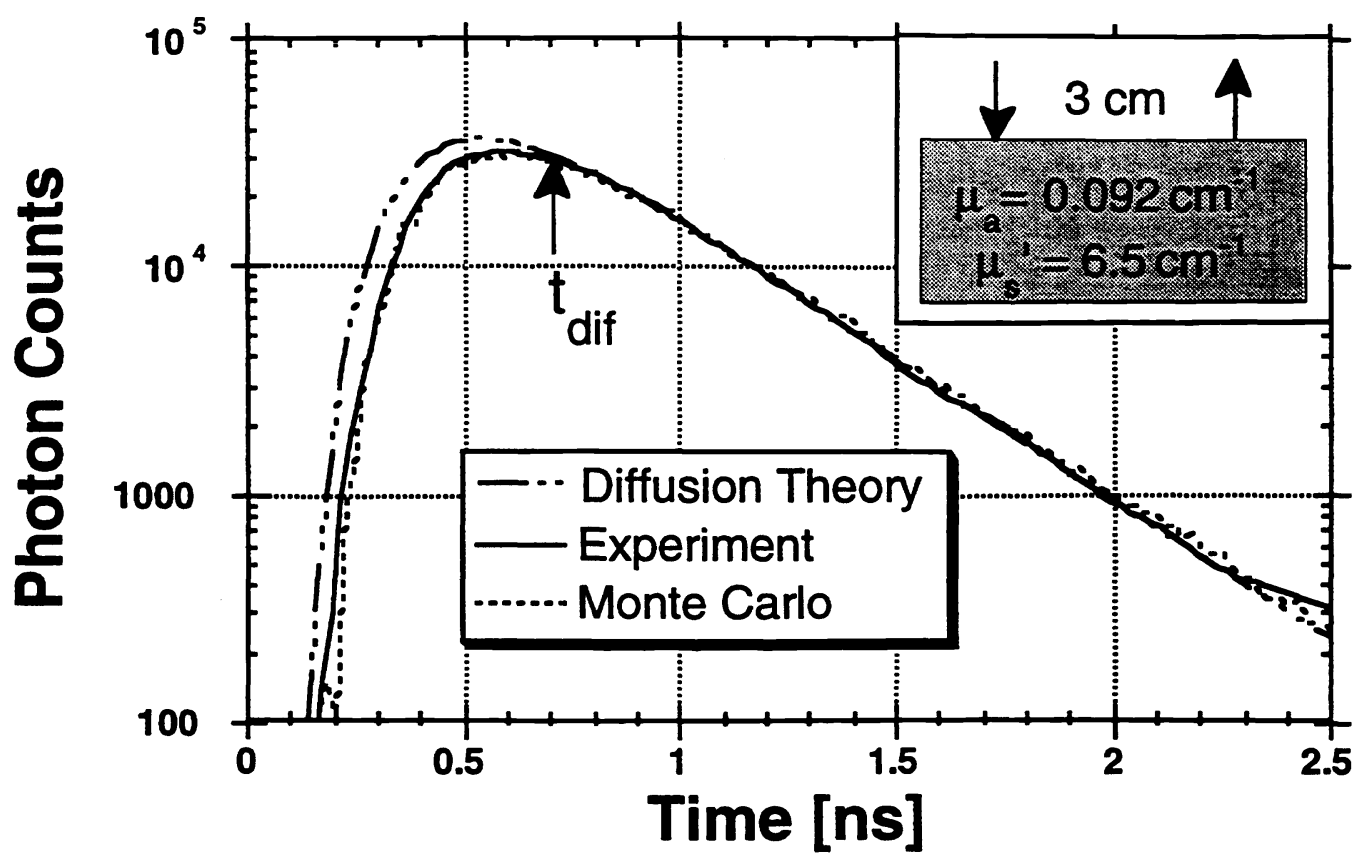

Fig.1: Comparison of time resolved reflectance experiment, diffusion theory and Monte Carlo simulation.

A much faster way to extract optical properties from an experimental data set is to fit simple analytical expressions found through diffusion theory 17 . Light propagation is treated here as a diffusion process. However this theory partially fails to describe the photon transport accurately, as shown in figure 1 . On can observe that after a certain time $t_{\text {dif }}$, the diffusion theory agrees well with the Monte Carlo simulation and experiment, while at early times the diffusion theory suggests a too strong signal which starts rising to early.

Knowing that diffusion theory gives accurate results at late times, the question is what optical properties can we determine by using just this part of the time resolved reflectance? The expression for the reflectance measured at a distance $r$ at time $t$ is given by (for a more detailed derivation see [17]):

$$
R(r, t)=(4 \pi c D)^{-3 / 2} t^{-5 / 2} \exp \left(\frac{r^{2}+\left(1 / \mu_{s}^{\prime}\right)^{2}}{4 c D} \frac{1}{t}\right) \quad \exp \left(-\mu_{a} c t\right)
$$

where $\mu_{s}$ ' is the reduced scattering coefficient, $D=\left[3 \mu_{a}\left(\mu_{a}+\mu_{s}{ }^{\prime}\right)\right]^{-1}$ the diffusion constant and $c$ the speed of light in the medium. $R(r, t)$ essentially consist of an amplitude factor and two 
exponentials. One exponential depends on $1 / t$ and the other one on $t$. For large times the first exponential goes to 1 and the second exponential term, which contains $\left(\mu_{\mathrm{a}} \mathrm{ct}\right)$ dominates. Thus $\mu_{\mathrm{a}}$ can be found from a fit to the tail of the curve. Eq. (1) can be linearized in $\mu_{\mathrm{a}}$ and $\mu_{\mathrm{s}}$ ' by taking the natural logarithm of $R$ and assuming $\mu_{\mathrm{s}}{ }^{\prime}>>\mu_{\mathrm{a}}$ :

$$
\ln (R(r, t))=\text { const }-5 / 2 \ln (t)-\frac{\left(r^{2}+1 / \mu_{s}{ }^{2}\right) 3 \mu_{a} \mu_{s}^{\prime}}{4 c} \frac{1}{t}+\mu_{a} c t
$$

This provides a simple and fast linear fitting algorithm.

Why does diffusion theory not work at early times? This can be understood by the fact that the photons have to travel some distance or time within the tissue before they loose their original directionality and become really diffuse (randomized). Furthermore it has to be noted that the expression given in eq.(2) is derived for a semi-infinite medium with the so called zero boundary condition. Using a more appropriate boundary condition leads to better results at early times, however for the price of complexity. A linearization of eq.(2) in $\mu_{\mathrm{a}}$ is not possible anymore and more advanced and more time consuming fitting algorithms are required. As a rule of thumb we found that for the optical properties of interest, the diffusion theory with zero boundary condition can be considered accurate after $\sim 400-700$ ps.

It can be concluded that even though diffusion theory is not as accurate as Monte Carlo simulations in the overall description of the time resolved reflectance, it can be used very well for an accurate determination of the absorption coefficient of a homogenous medium. The deviation of the diffusion theory from the experimental data occurs only at early times, and has little effect on the determination of $\mu_{\mathrm{a}}$. The absorption coefficient controls the shape of the late part of the impulse response, were diffusion theory shows good agreement with the experiment and Monte Carlo simulations. Monte Carlo simulations are more accurate, but take much more time to finish. Thus they may not be used in partical fitting algorithms. However they may well be used as "gold standards", to check the accuracy of diffusion theory.

\subsection{Layered Tissue Structures}

When time resolve reflectance measurements are made on the head, the light injected into the tissue does not encounter a homogeneous medium. The brain is actually encapsulated by layers of skin, skull and the meninges. The questions arise: "How do these layers influence the time resolved reflectance measurements ?" and "Can the simple linear fitting routine based on eq.(3) still be applied?"

Before complex multi-layer tissues are considered, simpler two layer structures are investigated. As a first example figure 2 displays a time resolved Monte Carlo simulations for the following systems: Curve $I$ is the results for a homogenous medium, with a low absorption coefficient of $0.01 \mathrm{~cm}^{-1}$. Curve II belongs to a homogenous medium with a high absorption coefficient of $0.2 \mathrm{~cm}^{-1}$. Inbetween the reflectance for a layered system with an $8 \mathrm{~mm}$ layer of the high absorbing tissue on top of the low absorbing tissue is shown. The time response of the layered tissue follows in the early time the curve II for the homogenous medium with high absorption. 


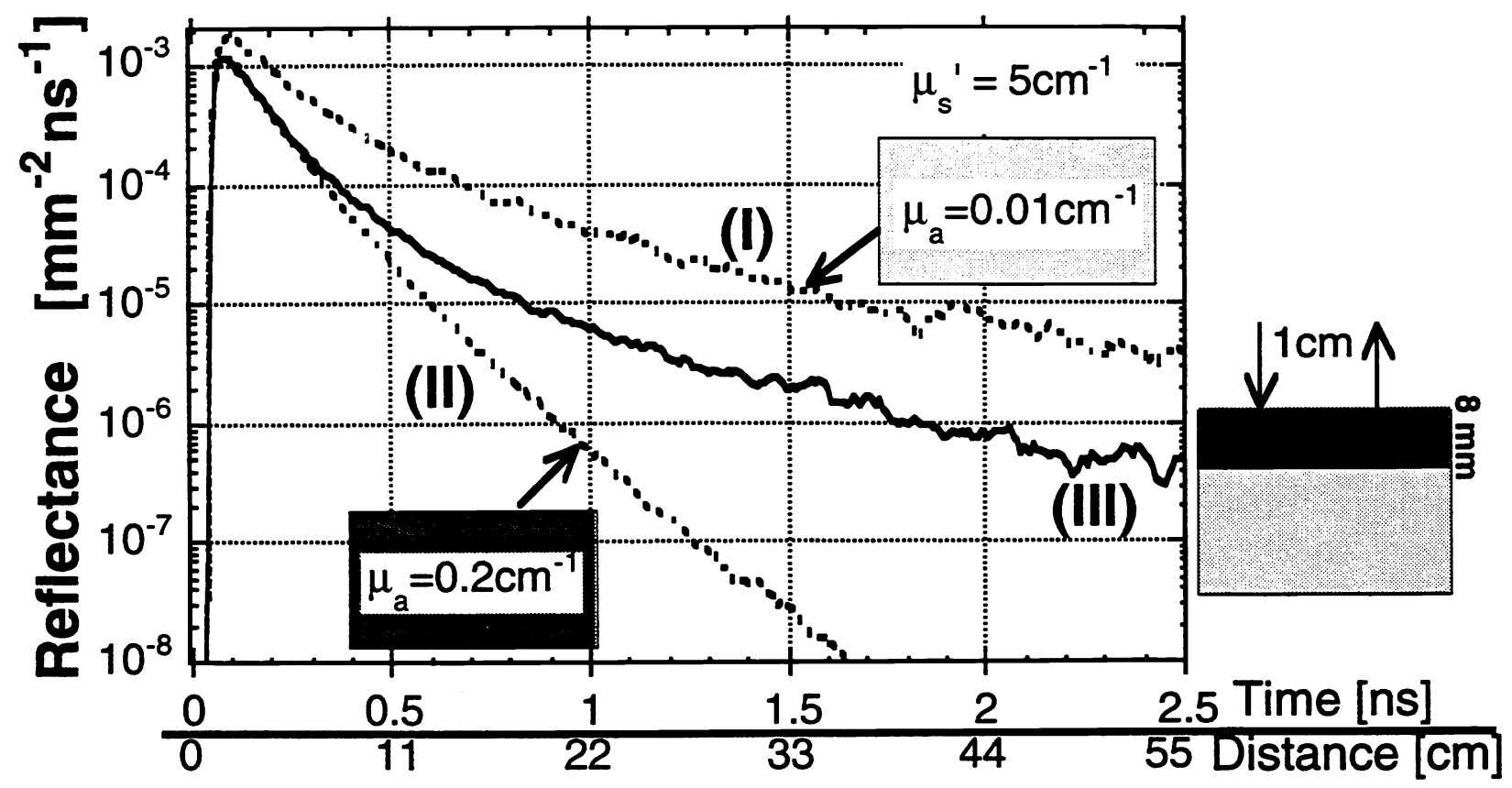

Fig.2: Monte Carlo simulation of a layered system with a high absorbing material on top of a low absorbing material.

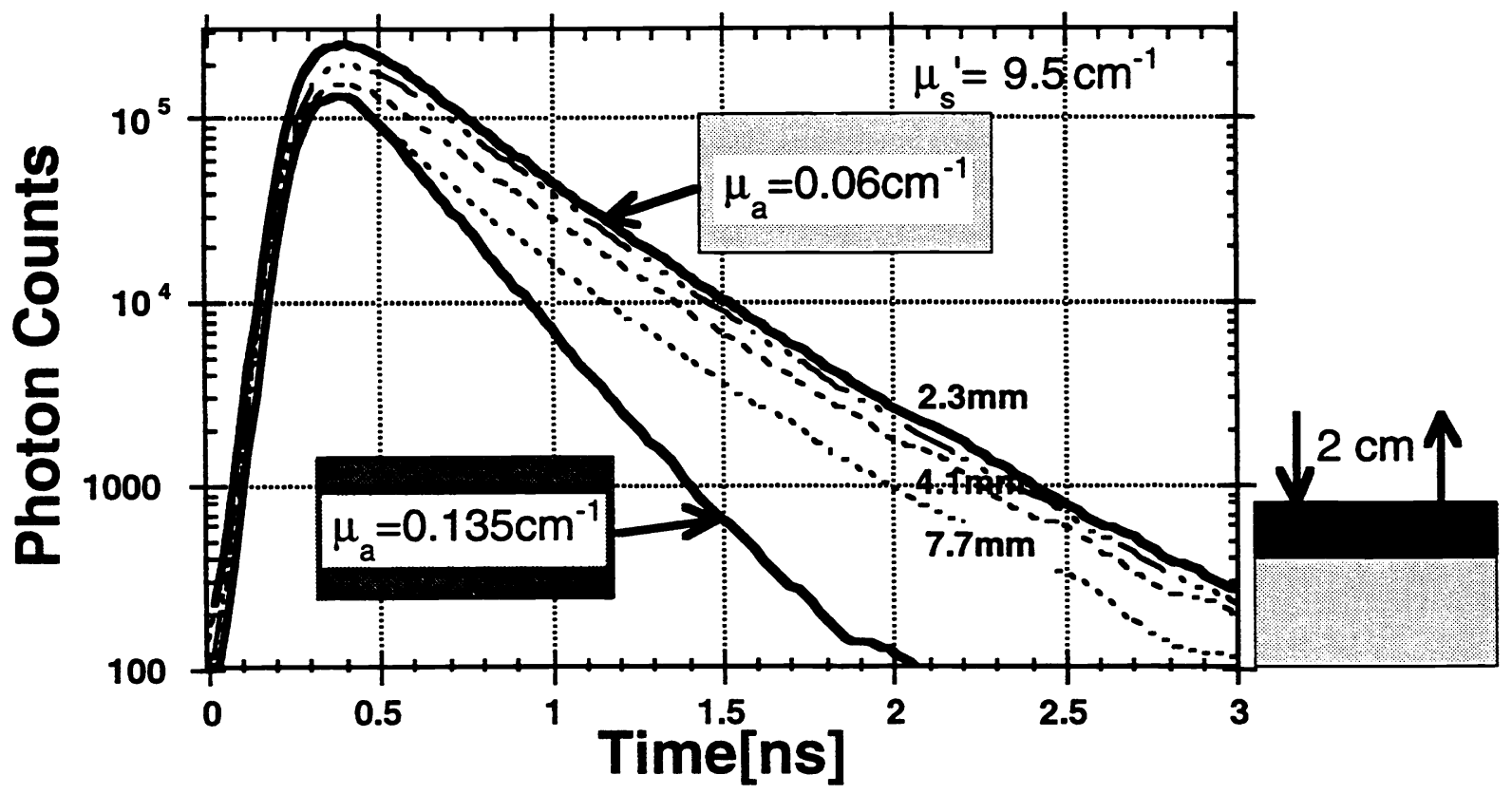

Fig.3: Experiments on layered gel phantom with a layer of high absorbing gel on top of a low absorbing gel. The values labeled near the curves are indicate the thicknesses of the top layer.

The photons travel only in the upper layer and are not influenced by the lower layer. After roughly 400 ps curve II and III start to deviate. This indicates that photons which have reached the lower layer contribute to the signal. After $\sim 1 \mathrm{~ns}$ the curve of the layered medium becomes parallel to the 
curve I of the homogenous medium with the optical properties of the lower layer. As shown earlier, the absorption coefficient of a tissue is only determined by the slope of the late part of the time resolved reflectance. Thus a fit of diffusion theory (eq.(3)) to curve I (homogenous medium) and curve II (layered medium) yields the same $\mu_{\mathrm{a}}$. This suggests, that one can measure through the upper layer the absorption coefficient $\mu_{\mathrm{a}}$ of the hidden tissue.

This might be surprising, on the first view. However, if one looks at the numbers which are added under the time scale one can see that after $1 \mathrm{~ns}$ the photons actually have traveled $\sim 22 \mathrm{~cm}$ in the tissue. This means that by far most of the time a photon propagates, it spends in the lower layer. The $8 \mathrm{~mm}$ thick upper layer becomes optically thinner and thinner the more time elapses.

The results of the simulations were also tested experimentally on layered tissue phantoms, made out of collagen gels. Polystyrene spheres with a diameter of $207 \mathrm{~nm}$ were added to the gel to introduce scatterers into the medium. India ink in different concentrations served as absorber. Gels with different optical properties were stacked on top of each other to yield a layered tissue structure. The findings from Monte Carlo simulations could be confirmed as shown in figure 3 . Through the upper layer one can measure the absorption coefficient of the underlying medium, by fitting the late part of the time resolved reflectance. Different thicknesses of the upper layer seem only to affect the amplitude of the signal, but not the determination of the absorption coefficient of the lower layer.

Simulating next the skull on top of the brain, the case was considered, where a low absorbing layer is on top of a high absorbing tissue. Figure 4 shows the corresponding Monte Carlo simulations. The curves of the homogenous medium ar erepresented by the upper and lower curve, respectively. The curves for the layered tissue structures with different thicknesses of the upper layer are displayed inbetween. Very similar to fig.3, after a certain time, the tail of the time resolved reflectances are dominated by the absorption properties of the lower layer. Different thicknesses of the upper layer only result in an amplitude shift and a delay of the time when the tails of the curves become parallel. The results from the simulations are confirmed experimentally with a layered gel tissue phantom (Fig.5).

Until now the scattering coefficients $\mu_{\mathrm{s}}$ ' in both layers were the same. However one might argue that if the scattering coefficient in the upper layer is higher than in the lower layer, photons are kept longer in the upper layer an thus the influence of the upper layer is increased. Figure 6 shows results from time resolved Monte Carlo simulations. The lower curve corresponds to a homogenous medium with a sčattering coefficient of $\mu_{\mathrm{s}}{ }^{\prime}=5 \mathrm{~cm}^{-1}$ and an absorption coefficient of $\mu_{\mathrm{a}}=0.2 \mathrm{~cm}^{-1}$. Now a $4 \mathrm{~mm}$ lower absorbing layer, with the same $\mu_{\mathrm{s}}$ ' is added on top. The amplitude is increased, however as already discussed after a certain time the tail of the two layer system is parallel to the one of the homogenous medium. Next the scattering coefficient of the upper layer is increased three times, which is quite large for biological tissues. This increase in scattering leads to a smoothening of the peak in the early part. However, in this example no significant increase in the time when the tail of the curves become parallel can be seen. Thus also in this case the absorption coefficient of the lower layer can be retrieved. 


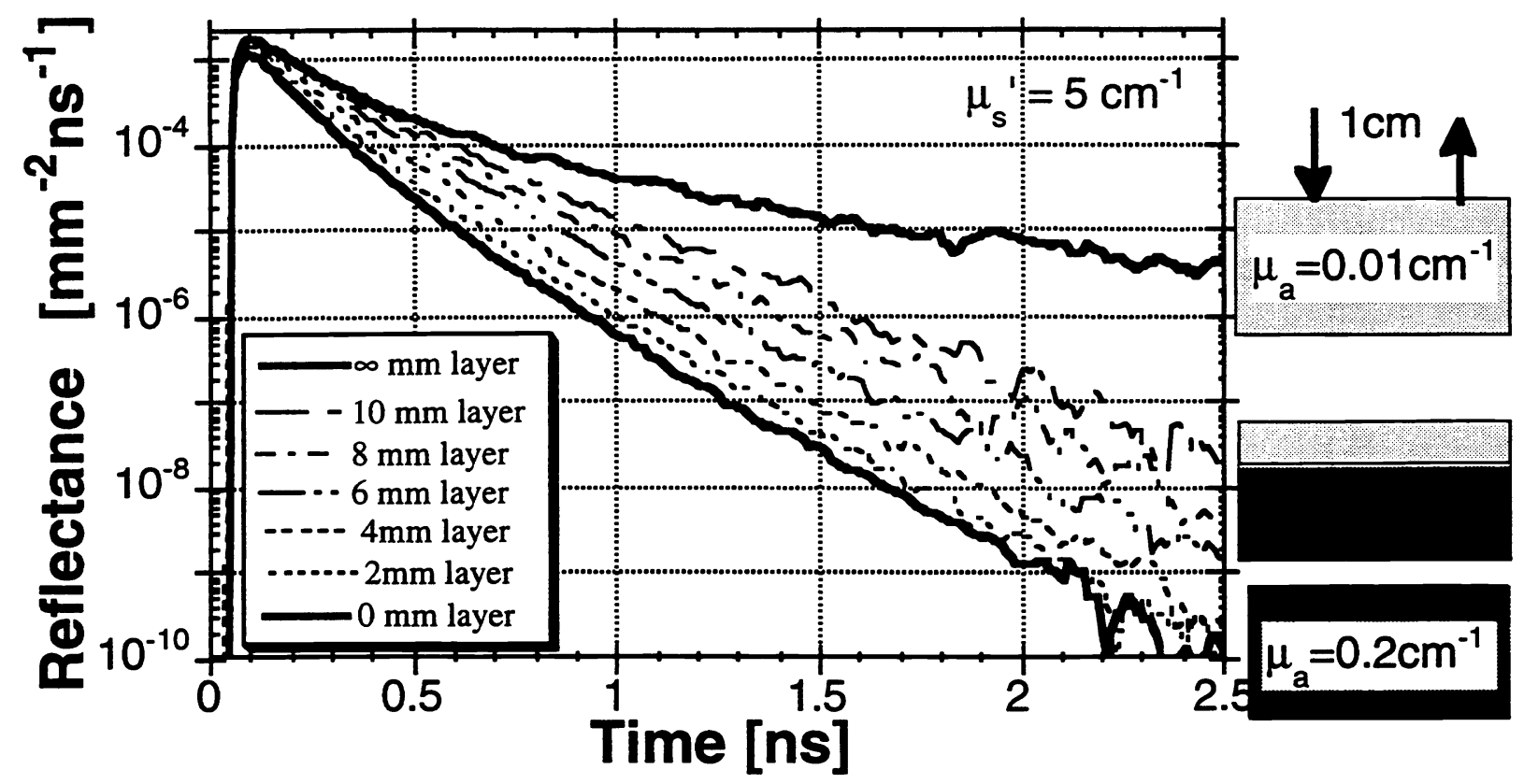

Fig.4: Monte Carlo simulation of a tissue structures with a layer of low absorbing material on top of a high absorbing material.

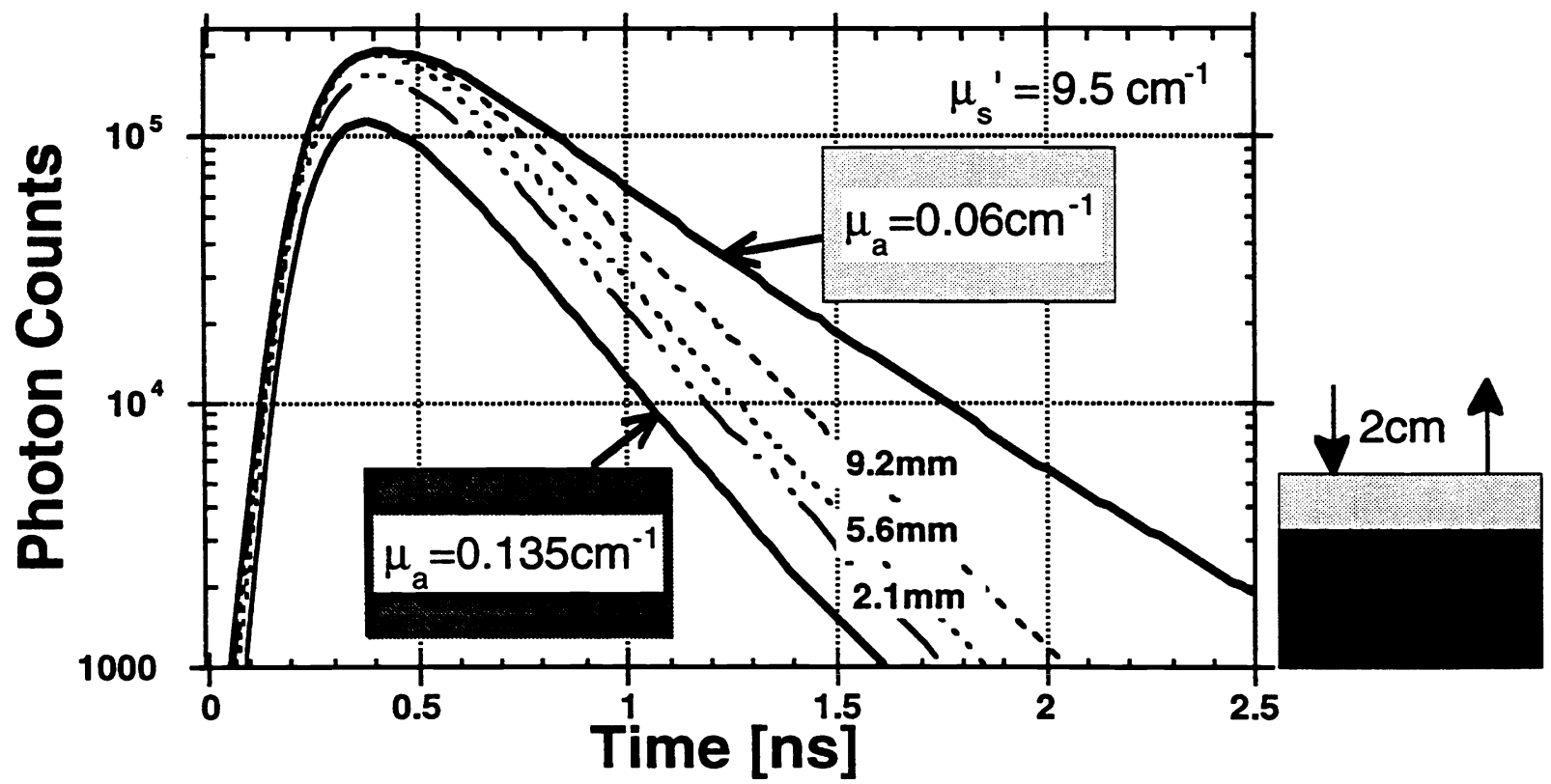

Fig.5: Experiments on layered gel phantoms with a layer of low absorbing gel on top of a high absorbing gel. The values labeled along the dashed curves indicate the thicknesses of the top layer. 


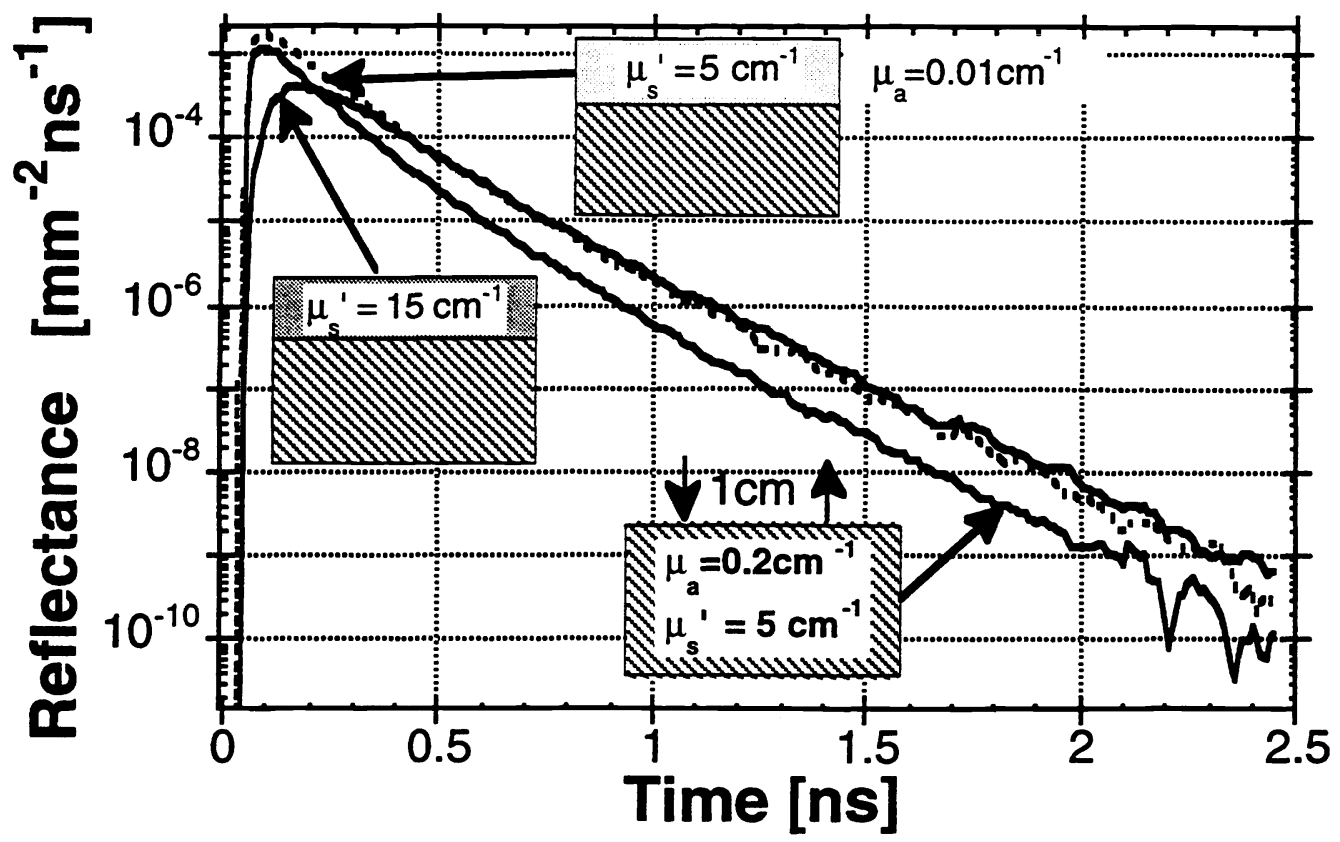

Fig.6: Monte Carlo simulation of a two layer tissue with varying absorption and scattering coefficients.

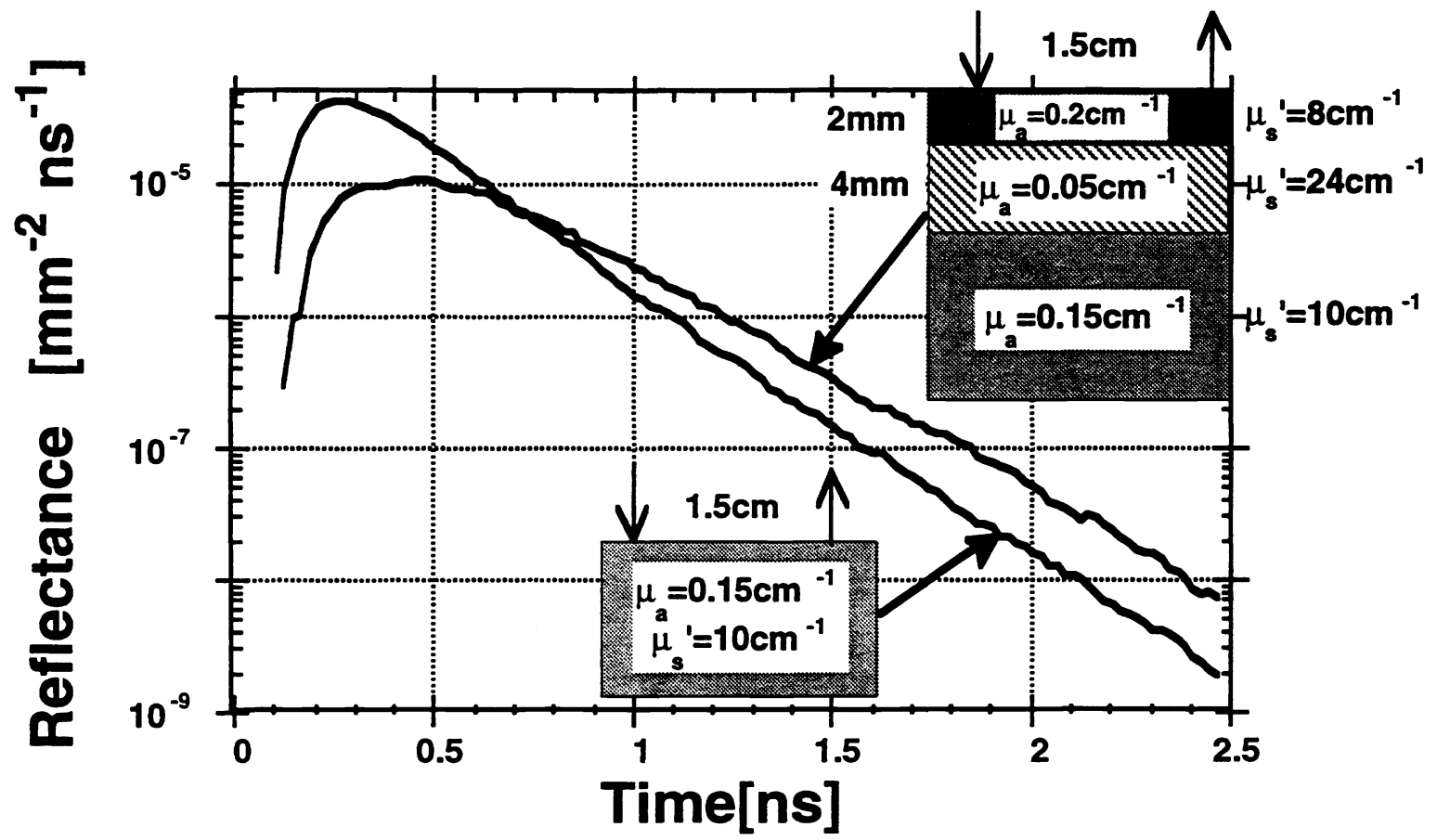

Fig.7: Monte Carlo simulation of a multi layer tissue with varying scattering and absorption coefficient, as labeled in the graph.

Figure 7 finally shows a simulation of a three layer system. All three layers differ in absorption and scattering coefficient which are similar to typical values for skin, skull, and brain tissues in the near infrared. The second curve corresponds to a homogenous medium with the 
optical properties of the underlying medium. After $1.5 \mathrm{~ns}$, the tail of the two curves become parallel, which once again confirms that the lowest layer in a layered system determines in general the late part of the time resolved reflectance. By fitting diffusion theory (eq.3) to this part of the experimental data one can obtain the absorption coefficient $\mu_{\mathrm{a}}$ of the underlying medium. Thus the absorption coefficient $\mu_{\mathrm{a}}$ of the brain tissue is accessible through the skin, skull, and meninges! Over eq. $(1 \mathrm{a}, \mathrm{b}) \mu_{\mathrm{a}}$ is connected to the blood oxygenation in the brain.

\section{SUMMARY}

This work was motivated by the fact that measuring the blood oxygenation in the brain is in particular important on newborn intensive care units as well as during and after cardiac surgery. Current devices, based on pure light absorption measurements, can only detect changes in blood oxygenation but fail to give absolute values for the brain blood oxygenation. This limitation is due to the lack of knowledge of the path length $L$ of the photons reflected from the head. Thus it is essential to measure the absorption coefficient $\mu_{\mathrm{a}}$, this is absorbance per unit path length. In this work it was proposed to use time resolved reflectance measurements, rather than a continuous light source to probe the tissues. In this technique a short light pulse $(\sim 50 \mathrm{ps})$ is injected into the tissue and the time resolved reflectance is measured at a distance of $\sim 1-3 \mathrm{~cm}$.

In studying how the absorption coefficient $\mu_{\mathrm{a}}$ can be extracted from time resolved reflectance measurements, two cases were discussed: In homogenous media, diffusion theory, even so incorrect at earlier times can be used to determine accurately the absorption coefficients. The shape of the reflectance curve for times larger than roughly $400-700 \mathrm{ps}$, when diffusion theory is accurate, provides the necessary information. In layered media, the properties of the upper layer only influence the time resolved reflectance at early times. The late part of the impulse response yields information about the absorption coefficient $\mu_{\mathrm{a}}$ of the lowest layer. The absorption coefficient is an indicator for the blood oxygenation. More discussion on the determination of the blood oxygenation from the measured $\mu_{\mathrm{a}}$ values, will be given in the next paper ${ }^{14}$.

\section{ACKNOWLEDGMENT}

This work was supported in part by the Department of Energy and the Robert A. Welch Foundation.

\section{REFERENCES}

[1] Pape K.E., Wigglesworth J.S., "Haemorrhage ischaemia and the prenatal brain", Heinemann Medical Books (London) for Spastics Int. Med.Publ. (1979)

[2] Stewart A.L., Thorburn R.J., Hope P.L., Goldsmith M., Lipscomb A.P., Reynolds E.O.R.,

"Ultrasound appearance of the brain in very preterm infants and neuro-developmental outcome at 18 months of age", Archives of Diseasa in Childhood 58, 598 (1983)

[3] Benaron D.A., Benitz W.E., Ariagno R.L., Stevenson D.K.,"Noninvasive Methods for Estimating In Vivo Oxygenation", Clinical Pediatrics 31, 258 (1992) 
[4] Cope M., Delpy D.T., "System for long-term measurement of cerebral blood and tissue oxygenation on newborn infants by near infrared transillumination", Medical and Biological Engineering and Computing 26, 289 (1988).

[5] Jöbsis F.F., "Noninvasiv, Infrared Monitoring of Cerebral and Myocardial Oxygen Sufficiency and Circulatory Parameters", Sciene 19, 1264 (1977)

[6] Wray S., Cope M., Delpy D.T., Wyatt J.S., Reynolds E., "Characterization of the near infrared absorption spectra of cytochrome aa3 and haemoglobin for the non-invasive monitoring of cerebral oxygenation",Biochemica et Biophysica Acta 933, 184 (1988)

[7] Thorniley M., Livera L., Wickramasinghe Y.,Spenccer S.A.,Rolfe P., "The non invasive monitoring of cerebral tissue oxygenation", Adv. in Exp. Med. Biol. 277, 323 (1990)

[8]Tamura T.,Eda H.,Takada M. ,Kubodera T., "New Instrument for Monitoring Hemoglobin Oxygenation", Adv. in Exp. Med. Biol. 248, 103-107 (1990)

[9] Chance B.,Leigh J.S.,Miyaka, H.,Smith D.S.,Niola D.S., Greenfeld R., Finander M.,Kaufmann K., Levy W., Young M., Cchen P., Yoshioka P., Boretsky R., "Comparison of time Resolved and Unresolved Measurements of Deoxyhemoglobin in Brain", Proc. National Academy of Science 85:4971 (1988)

[10] Benaron D.A., Gwiazdowski S.,Kurth C.D., "Optical path length of $754 \mathrm{~nm}$ and $816 \mathrm{~nm}$ light emitted into the head of infants", Proc. IEEE Eng. Med. Biol. 1990; 3, p.1117 [11] Duck F.A., "Physical Properties of Tissue", Academic Press, San Diego, Ca (1990) [12] Svaasand L.O., Ellingsen R., "Optical Properties of Human Brain",Photochem. and Photobiol. 38, 293 (1983)

[13] H. Liu, M. Miwa, B. Beauvoit, N.G. Wang, B.Chance, "Characterization of absorption and scattering properties of small volume biological samples using time-resolved spectroscopy", Analytical Biochemistry 213, 378-385 (1993).

[14] H. Liu, A.H. Hielscher, B. Beauvoit, L. Wang, S.L. Jacques, F.K. Tittel, B. Chance, "Determination of blood oxygenation in the brain by time resolved reflectance spectroscopy (II): Contribution of vascular absorption and tissue background absorption", see this Proceedings. [15] S.L. Jacques, L.H. Wang, A.H. Hielscher,'Time-Resolved Photon Propagation in Tissues" in Optical and Thermal Response of Laser Irradiated Tissue, editor A.J.Welch, M.van Gemert, Plenum Publishing Corp. New York, to be published in 1994 [16] I. Lux, L. Koblinger, Monte Carlo Particle Transport Methods: Neutron and Photon Calculations, CRC Press, Boca Raton, Florida, 1991.

[17] Patterson M.S., Chance B., Wilson B.C., "Time resolved reflectance and transmittance for the non-invasive measurement of tissue optical properties", Applied Optics 28, 2331 (1989) 